December - 2009

\title{
A Case Study of an International E-Learning Training Division: Meeting Objectives
}

\author{
Rory McGreal \\ Athabasca University, Canada
}

\begin{abstract}
This paper presents an evaluation of the work of the Commonwealth of Learning's (COL) eLearning with International Organisations (eLIO) section. Participants in the investigation included a representative sample of the learners $(N=15)$, their supervisors $(N=5)$, and the COL staff, including all of the eLIO staff $(N=10)$. The methodology consisted of an examination of all relevant documents, interviews that formed a learning history, and a sample survey. The investigation concluded that the eLIO achieved its goal of developing a distance learning model, and it met or exceeded identified objectives, with a high degree of satisfaction expressed by all participants. This included teaching +2000 satisfied learners; partnering with eight international organizations; achieving a $62 \%$ female participation rate and a high completion rate (75\%) in the courses provided; testing, piloting, and delivering two new elearning courses; conducting needs analyses; recruiting/training highly qualified tutors; monitoring; and using appropriate technologies. Shortcomings of the programmes include the lack of pre- and post-tests, little analysis of pricing structures, some unclear instructions (a need for plain English), unclear copyright licensing, only very limited use of available OER software, and the absence of a succession plan for the manager. Based on the high level of satisfaction among all participants, it was recommended that the section maintain its present work and address these shortcomings.
\end{abstract}

Keywords: Distance education; open learning; e-learning; online learning; elearning management

\section{Commonwealth of Learning's E-Learning for International Organisations Section}

The Commonwealth of Learning (COL) is an intergovernmental organisation created by the Commonwealth Heads of Government to encourage the development and sharing of open learning and distance education knowledge, resources, and technologies. The COL eLearning for International Organisations (eLIO) section provides technology-enhanced, customised training solutions for international organizations, such as the World Bank and UNESCO. Services include needs analyses, development of customized culture- and gender-sensitive training materials, and 
course delivery at a distance using expert tutors. Many international organizations, aware of COL's international reputation for distance education in the developing world, are contracting with the eLIO for their training needs. This paper provides an evaluation of this COL section and makes recommendations for improvements (Commonwealth of Learning, 2008h).

\section{Significance of the Investigation}

The increased availability of computers and the Internet has made possible the delivery of training to populations, particularly those in developing countries who previously could only be reached by using traditional classroom-based teaching approaches or by using often ineffective correspondence courses, which have high dropout rates. In order to ensure the effective adoption of these new technologies for learning, open access to case studies and other investigations of these distance education initiatives is important. These can serve as useful guides to others who are planning similar or complementary implementations. Knowledge of the research on successful or even unsuccessful training practices can help others to better plan their own systems (Cruickshank, 1990).

The rapid advance of technology combined with the ever-increasing need for skilled professionals with technological and English language skills must be addressed. How to reach these professionals in widely dispersed international organizations, especially employees in developing countries, remains a serious concern. As researchers, we have an obligation to study programme implementations that address this problem and to provide an understanding of the difficulties and of the successes of the real-world implementations.

For example, distance educators must evaluate the technologies that they choose for delivery. Which technologies are appropriate given the target population and their degree of access to computers and the Internet? As far back as 1983, Clark determined that the technology was neutral, conferring no learning benefits regardless of the media used.

More closely related to this investigation is an examination of the role of the administration in an implementation project and in the training system as a whole. In recent times there has been a (perhaps necessary) focus on the learner as the centre of the process. This has often led to a deemphasis on the training system as a whole and on the part that it plays. This study focuses on the administration of the training system.

As a case study, this investigation may shed some light on what is needed for a successful implementation of distance training internationally. Flyberg (2006) reminds us that our understanding may be strengthened by the execution of a greater number of good case studies. Mathur and Oliver's (2007) case study of an international blended learning programme for developing countries sheds light on the need for technological knowledge and also on the problems and pitfalls of collaboration between organizations.

Oliveira, Cortimiglia, and Marques' (2003) case study of a data technology distance education course implementation looked at the technology requirements, financial results, and market 
possibilities. They concluded that their web course was capable of reaching remote areas and the instructional contents were capable of delivering professional qualifications.

Hills (1999), in her case study of Lloyd's bank training, focused on the organizational culture and its effect on training. She concluded that a learning culture is needed for the exploitation of multimedia for training. A faculty learning community was the subject of Long, Janas, and Kay, et al.’s case study (2009) of a small college online learning implementation.

Failed implementations are also worthy of our attention, especially when the implementation is similar to one under investigation. Nchindila (2007) investigated the conditions for the success of the writing process in English using online mentoring in the workplace, concluding that in this case study the implementation was evaluated as not successful, based on questionnaires for the students and mentors.

\section{Outcomes and Outputs and Related Success Indicators}

The original outcomes and outputs were as stated in Table 1.

\section{Activities Implemented}

The eLIO team developed and delivered technology-mediated distance learning programmes with international organizations in different regions around the world. They formed partnerships with international organizations in order to accomplish this. The learning materials were developed and customized for each organization to promote the learning of relevant occupational skills, based on an analysis of the learners' needs and on the capacity of the technological infrastructure available. Qualified tutors were engaged and trained in order to facilitate the delivery of the programmes. Seven international partners participated as partners in eLIO programmes. 
Table 1

Planned Outcomes and Outputs

\section{Outcomes}

- Development and delivery of technology-mediated distance learning programmes with international organisations to enable access to learning by a dispersed and diverse international workforce.

- By 2009, at least 6 major international organisations active in delivering the Millennium Development Goals (MDGs) have formed a partnership with COL in customising gender- and culture-sensitive workplace eLearning programmes.

- An additional 2,000 adult learners (a 6 to 4 ratio of women and men) will be trained by 2009.

- At least two new contextually relevant sets of ODL/ICT-based learning materials for occupational skills development created and each is taken up by at least two major institutions.

- Did the COL eLIO achieve its goals through the activities posited? Activities:

- Actions to achieve the outputs

1. Analyse and understand the learners' needs, learning objectives and outcomes, the learning environment and technology infrastructure of the organizations.

2. Design courses and develop materials through customization.

3. Test all training materials and delivery designs

- Build responsive infrastructure and clear processes for course production and presentation. This includes

- $\quad$ preparing storyboards/templates;

- using robust, affordable and user-friendly technology and media;

- recruiting and maintaining a cadre of trained and committed tutors;

- using OERs software as far as possible.

- Monitoring Data

a. pilot evaluation reports

b. monthly progress reports

c. tutor listservs ${ }^{\circledR}$

d. end of course evaluation feedback

e. programme evaluation studies

\section{Outputs}

- number of participants

- number of organizations served

- number of regions served

- revenue

\section{Success Indicators}

+2000 by 2009

6

not stated

self sustaining by 2009

\section{Limitations of the Study}

This plan is limited to an evaluation of the activities of the COL eLIO and does not address the wider issues associated with the COL organization. The evaluation is for this particular section only, and it is recognized that the external validity or generalisability is limited. Reliability is always an estimate. It was addressed by triangulating the data collected using at least three different programme case studies, documents, a survey, and interviews with a random sample of 
participants, COL eLIO staff, and others using similar questions and interviewing techniques. Through this triangulation a hybrid was attempted between quantitative and qualitative methods (See Leedy, 1993).

The validity of the evaluation conclusions, in this case, cannot be assured by a control group. There is often a multi-group threat to the validity of an evaluation when comparing different groups taking the same programme. As this evaluation did not compare the outcomes of the different groups, but rather considered the section's activities overall, including the programmes with different organizations, this was not a problem.

\section{Method}

The evaluation was conducted using triangulated data collected from several different pilot and programme evaluations, published papers, a simple survey instrument, and other documents as well as interviews with a random sample of participants (student, partners, and tutors). The COL staff and a sampling of others were interviewed using similar questions and interviewing techniques. These included seven COL staff members, five tutors, three partners, and 10 learners. The interviews were collated into a learning history document.

The information collected consisted of a large corpus of documents, including previous project evaluations supplied by the COL eLIO, the COL, and other relevant websites' published articles. Documents were obtained directly from the eLIO and from Internet searches. The documentation was positive in the extreme with none pointing to serious or even minor criticisms. At first this seemed suspicious but was borne out in the triangulation with the interviews and questionnaires.

In addition, interviews were conducted with the COL eLIO staff, other COL personnel, and a small sample of participants in the COL eLIO projects. A random sample of representatives from three of the eight participating organizations was contacted and interviewed. A random sample of tutors (five from a list of 20) was also contacted and interviewed. Thirty students were randomly selected and asked to participate in an online survey using Survey Monkey. Ten responded and one agreed to be interviewed by telephone. The random samples were elicited using the application available at Random.com. The participants in each group were given a number and then the numbers were input into the random.com application, which randomized the total and picked a sample. The participants contacted were sent a consent agreement.

A list of questions was used as the main instrument for the interviews. Other supplementary questions were used as they emerged out of the discussion with participants. The analysis included an examination of the question responses from which data was collected. This data from the quantitative questionnaires was analysed statistically (See Nunan, 1992).

These surveys were triangulated with the interviews and documentation. The reliance on a variety of sources in this investigation is supported by Yin (1994), who argued that one of the benefits of a case study is that it depends on multiple sources of data as evidence. 
The interviews were conducted either by telephone or in a face-to-face format in June 2008. The information was collected, converted into a learning history format, and analysed for this report. A learning history methodology provides organisations with a special approach to learning from the experiences of the participants. In the right-hand column, relevant observations are described by the people who took part. The participants are quoted directly, and their statements are placed in a category defined by their role to increase anonymity. The researcher then attempts to weave the words into a coherent story based on the information provided, including facts, results, and assumptions of the participants. This methodology is based on approaches and techniques in a variety of fields, including organizational learning (See Roth \& Kleiner, no date).

This evaluation consists of a summative assessment of the outputs and achievements of the COL eLIO for formative input into planning future programme implementations as well as for expanding partnerships and for increasing the efficiency and effectiveness of the sector. The key performance indicators - output and outcomes - have been identified from COL documents and in consultation with the COL eLIO representative. These include information on the organizations served and on the participants in the programmes delivered (See Table 1). Appropriate documentation and access to interviewees was made available to the evaluator, who developed a strategy for analysing the data provided. Indicators included data on the growth of the initiative (e.g., number of courses created and delivered, number of learners, learner achievement, engagement of technology, lessons learned, threats \& opportunities, expansion, gender equity, etc.).

This evaluation can be used by COL to inform its planning processes and to report on activities to COL stakeholders.

\section{Findings}

A 6R assessment criteria (relevance, results, reach, regions, resources, and relationships) was used. These criteria were triangulated with the documentation and survey results.

\section{Relevance}

There was a consensus among the COL staff that the eLIO is marginalized or "peripheral" primarily because it is the only section of COL that has been mandated to be self-supporting. They note that the eLIO, in fact, contributes significantly to the core mandate of COL as expressed in the COL Three Year Plan (Commonwealth of Learning, 2006). The eLIO's relevance to COL is something more than revenue generation.There is a strong alignment of its goals in serving international organizations with those of COL in increasing capacity and demonstrating the effectiveness of open and distance learning (ODL). It strives to serve as a successful example of the use of technology for ODL in developing countries, including the Commonwealth. The section is responsible for adding to the reputation of COL as a professional organization that serves the developing world by using the best approaches and techniques in DE. As an organisation that works with governments at the policy level for human resource 
development, COL's reputation is enhanced when the eLIO, at the grassroots level, demonstrates the viability, effectiveness, and sustainability of international ODL. As one interviewee noted, COL "needs something to crow about."

\section{Unexpected Outcomes}

The eLIO has also demonstrated its relevance by its actions because other opportunities have come to COL as a result of eLIO's reputation for delivering ODL. UNICEF is interested in a wider opportunity for COL in HR development (possibly $\$ 1.9$ million) along with a major capacity building project for South African civil servants. These opportunities have come to COL by word-of-mouth. Without advertising, the eLIO programmes have built viable relationships and have increased COL's visibility among international organisations, particularly major funders.

\section{Results}

The results to be measured include in the first instance the stated intended results of the eLIO section as per the COL strategic plan. They are listed below along with information on the success of each.

\section{Goal}

Development and delivery of technology-mediated distance learning programmes with international organisations to enable access to learning by a dispersed and diverse international workforce.

Results

Successful: The eLIO developed and delivered several distance learning programmes to international organizations with widely dispersed and diverse international workforces.

\section{Goal}

By 2009, at least six major international organisations active in delivering the Millennium Development Goals (MDGs) have formed a partnership with COL in customising gender- and culture-sensitive workplace eLearning programmes.

\section{Results}

The eLIO surpassed this goal, partnering with eight international organizations active in delivering e-learning that support MDGs. These have been generally described as being sensitive to gender and cultural issues in the workplace. The following organizations participated as partners with eLIO:

1. World Health Organisation,

2. UN High Commissioner for Refugees,

3. International Labour Organisation,

4. International Federation of the Red Cross and Red Crescent Societies,

5. The Joint UN co-sponsorships for HIV/AIDS, 
6. World Bank,

7. UNICEF, India Country Office,

8. Office of Internal Oversight Services (OIOS).

Goal

An additional 2,000 adult learners (a 6 to 4 ratio of women and men) will be trained by 2009.

Results

1,766 adult learners (62\% female) have received training from the eLIO section. This number increased to more than 3000 in 2009.

Goal

At least two new contextually relevant sets of ODL/ICT-based learning materials for occupational skills development created and each is taken up by at least two major institutions.

Results

Two new elearning courses have been developed, adapting the materials and contextualizing them to different organizations.

\section{Goal}

Did the COL eLIO achieve its goals through the activities posited?

a. Analyse and understand the learners' needs, the learning objectives and outcomes, the learning environment, and the technology infrastructure of the organizations

Results

Needs analyses were conducted with the partner organisations, taking into account the learning environment and the technological infrastructure. Adjustments to the courses were made based on these analyses; for example, deadlines and examination schedules were changed, CDRoms were used for distribution, and email was used rather than more complex applications on the Internet.

b. Design courses and develop materials through customization

Results

Six courses were adapted and delivered specifically for each organization, taking into account their different cultures and learning environments. All partners interviewed agreed that this was done very well and according to their needs.

c. Test all training materials and delivery designs

i. build responsive infrastructure and clear processes for course production and presentation

ii. prepare storyboards/templates

Results

One partner reported on the difference between her organization's approach to instructional design and that of eLIO, specifically referring to eLIO's change in approach to accommodate her 
organization's wishes. This included adopting storyboards and templates as part of the learning design process.

iii. use robust, affordable, and user-friendly technology and media

Results

The eLIO course designers deliberately focused on low technology solutions in order to ensure that the course would be robust, userfriendly, affordable, and, most importantly, accessible by the learners dispersed around the world in developing countries. This included the distribution of CDRoms for the course materials and the use of simple email for communications.

iv. recruit and maintain a cadre of trained and committed tutors

\section{Results}

The eLIO recruited and maintained a cadre of trained and committed tutors. The partners and students unanimously agreed that the tutors were indispensable and the most important part of the programme.

v. use OER software as far as possible

Results

No open educational resource (OER) software was used. The project was limited by the proprietary software available in the partner organizations. For example, MS Office, including MS Word, was a standard that was known and used by the participants.

\section{d. Monitoring Data}

i. pilot evaluation reports

Results

A selection of pilot evaluation reports was made available for this study (Commonwealth of Learning, 2003; Murphy, 2003; Ruhe, 2002; Shale, 2003)

ii. monthly progress reports

Results

Monthly progress reports supplied by the tutors in a standard format were a regular occurrence. A random sampling of these reports was provided to the evaluator.

iii. $\quad$ tutor listservs ${ }^{\circledR}$

Results

Tutors were able to communicate with their students, the administration, and the administrators using listservs. In addition a web conferencing application was made available for discussion groups.

iv. end of course evaluation feedback

Results

Learners were asked in the evaluations to provide their views on the course, their estimation of its success, and their attitude towards it. 
They were satisfied with their progress and very positive in their attitude towards the course and the tutors in particular. However, other than some very tenuous pre- and post-testing, there was no reliable testing of the achievement level of the learners. There are problems associated with administering such pre- and post-tests in a distributed environment. Nevertheless, as was pointed out by some of the participants, such a practical test should be an integral part of the programmes in order to show learners, partners, tutors, administrators, and outsiders that real progress in achievement levels was made through the course.

v. programme evaluation studies

Results

Programme evaluation studies were conducted and acted upon (Dunlop, 2005; John, 2005; Walker \& Dzakiria, 2005; Wilson, 2006 $\&$ 2007).

The following questions were also addressed.

Does the existing data gathering mechanism adequately cover the necessary information for analysis and reporting? Where are the gaps?

The evaluations of both the pilots and the full courses were conducted in a professional manner. The learner feedback, although difficult to arrange, was adequate for analysis with a return rate of $30 \%$ to $40 \%$. As previously stated, the only real gap in the learner evaluations is in the need for pre- and post- tests of achievement.

The time allotment by COL eLIO staff was set at $2 \%$ for initiative monitoring and evaluation. The staff budget was capped at $25 \%$ of the revenue generated. Was this goal achieved? By how much?

This refers to funding allotted for program monitoring and evaluation and it was achieved. 
Table 2

\section{Output Summary}

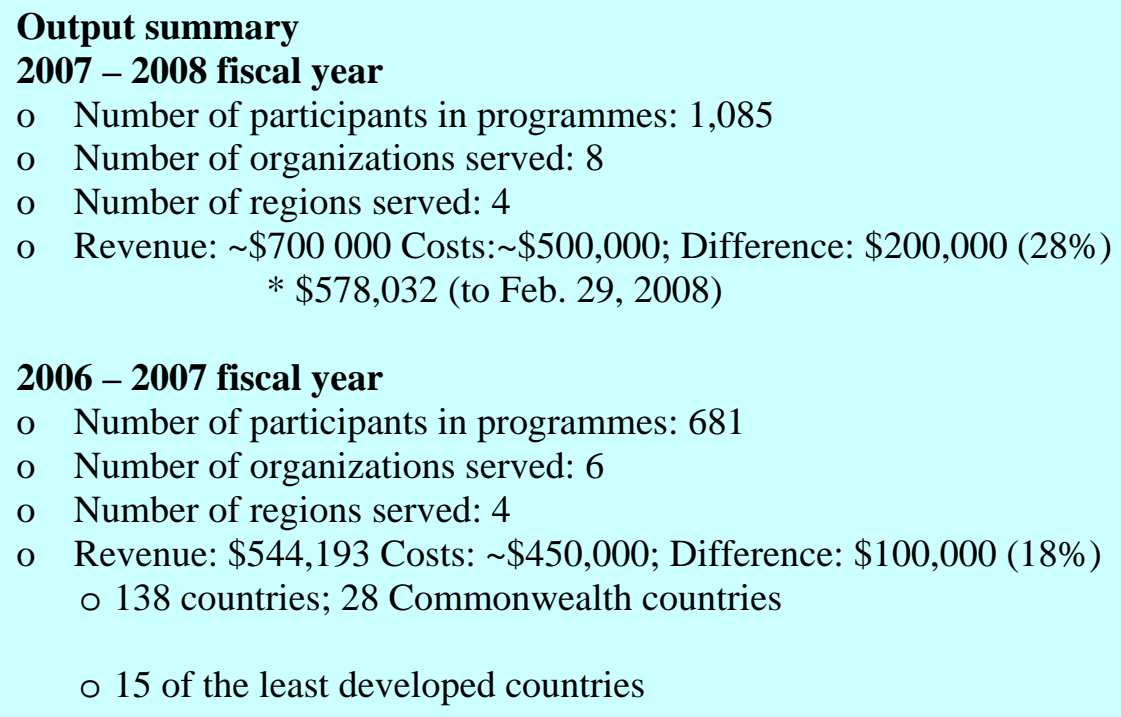

Besides these outcomes and outputs that were the goals of the eLIO as set out in the COL Three Year Plan, there were other results that need to be taken into account in any reasonable evaluation of the section. Foremost is the fact that more than 2,000 learners became more confident in their English writing skills. Many of these learners are either in Commonwealth countries (28), or they liaise with officials in Commonwealth countries. The student survey results showed that learners overwhelmingly felt that their writing and analytical skills had improved and that the course content was sufficient, clear and easy to understand, and appropriate for their organizations. They were particularly emphatic about the value of the tutorial system.

\section{Unexpected Opportunities}

Certainly, the unexpected opportunities with UNICEF and South Africa are among the unplanned results that can be used to further validate the section's work. The very high esteem for COL expressed by the partners is also important as their word-of-mouth advertising not only led to more partners within the UN system, but also to the above-named opportunities. There is real evidence of the success of the programme when partners renew their contracts while boasting of success to the others ("thrilled we found them!"). The customized curriculum for each organisation along with the personalized service was highly appreciated. The tutor system was also an important result that may not be apparent immediately. Tutors' empathy, flexibility, and personal skills, combined with a tight organizational structure, were critical components of the success of the programme. And it can be considered to be a key result of the programme itself when taken as a whole. 
Another significant result for the benefit of the partner organizations, which might also be of service to COL if implemented internally, was the programme's emphasis on standardizing communication practices around plain English writing. Partners felt that this helped them to standardize core functions among the learners, who operate using disparate cultural norms. More widely, as the courses spread to the different international agencies, it has become "a first," one of the rare examples of sharing resources among the different UN organizations.

On the negative side, the learners, the partners, and the tutors, while valuing the content highly, feel that there is room for improvement; for example, there is a need for clear section markers, a need for more contextualization, and a lack of variety in the examples. Tutors felt that the materials should be edited to reflect good practice in plain English.

\section{Reach}

Partners are convinced that online courses are the only viable way that they can reach their distributed staff for training. They particularly emphasise this need for ODL in developing countries and for staff who are traveling or who have fragmented work assignments. In this regard, they support the distribution of the courses on CD because of the erratic nature of Internet connectivity in many countries.

Tutors see a need for organizations to be more proactive in supporting ODL as a means of extending their training reach. They need to develop a culture of using DE. Tutors could be used to train those within the organisation in ODL skills. In addition, more courses on a wide range of training subjects could be developed and delivered.

Internally, staff members refer to the need for other COL sections to benefit from the expertise and skills of those in the eLIO section. They feel that the section should be reaching out to other COL sections that could use its expertise in capacity-building and human resource development. Reaching out can also be achieved successfully by making the most effective use of new technologies as they emerge. The section should experiment more with novel applications as access to them becomes more common among the partners.

\section{Regions}

Courses were delivered in four regions of the Commonwealth (Africa, South Asia, the Pacific, and the Caribbean). As previously mentioned, among the most significant developments arising from the activities of the eLIO is the opportunity for a contract to deliver training to civil servants in South Africa. Using Canadian tutors to train South Africans as ODL tutors could strengthen COL's core mandate for capacity-building. The different regions could also be strengthened by using the technology to create regional groupings of learners, so they can engage in peer-to-peer learning and teaching. 


\section{Resources}

The ambiguous position of the eLIO was referred to previously. COL's emphasis on extra budgetary financing has resulted in the section being perceived as different from other COL sections. The eLIO may not be considered as "core" by the COL board particularly because it serves many non-Commonwealth countries. The eLIO is perhaps both core and extraneous and is more useful to COL than it might be otherwise because from within the organisation it is able to provide an opening or a window to other international organizations involved in complementary activities. Therefore, eLIO can be seen as a resource.

The eLIO's emphasis on using simple technologies, while important for promoting access and supporting students, may not be the best strategy in the management and administration of its programmes. Staff members use applications like Survey Monkey for their surveys, so they are taking some advantage of the technologies available. The recent adoption of Basecamp for computer conferencing is another example. However, there are other open source applications that staff might experiment with to improve the management and the efficiency of the learning process. This experimentation with technologies could form part of an extended mandate for the section in the research and development of DE. For example, a customer relationship management (CRM) application may help to improve learner support and administrative efficiencies. Open source applications do exist (e.g. SugarCRM, Vtiger, Campware).

\section{The Learning Manager}

Many of the respondents commented specifically on the professionalism and competence of the eLIO staff, making specific reference to the learning manager. She was commended for turning her section from an "ugly duckling" to a "swan." Others refer to the section as being very "person-based." Others say that she runs a "tight ship" as an excellent organizer and administrator. Some wonder if the section would have a future if this particular learning manager was no longer running it, even suggesting that COL would have to review the section and perhaps re-organize it if she leaves.

\section{Discussion}

From the beginning, there has been debate about the role of the eLIO in promoting the organization, developing partnerships, raising funds, and providing support for ODL. An important development in the debate has been the evolving understanding of the section's role in supporting COL's overall policy direction and, hence, the section's alignment with the organization's goals. For example, the eLIO's efforts in delivering training to UN agencies have resulted in opportunities from UNICEF and possibly from the South African government for other COL sections. These opportunities can relate directly to COL's core goal of capacitybuilding in the Commonwealth.

After the last evaluation, COL priorities moved in the direction of extra budgetary funding for the 
eLIO. In the past, the role of COL has swung between supporting demonstration projects and providing limited funding of research. In the present three-year plan, COL has settled into a preference for intergovernmental relations and policy. This very brief recounting of the shifting forces that have shaped COL serves at least to signal the twists and turns of both COL's goals and management philosophy, which the eLIO learning manager has had to follow. Without an appreciation of these shifts, evaluating the successes and failures of the eLIO programme could have been a very frustrating and puzzling enterprise.

\section{Conclusions}

The eLIO achieved its intended results, yielding significant benefits in its principal outcomes and outputs. It developed a model for potential application in a variety of different (but possible) organizational and educational settings or environments with some important additions to the stock of open learning pedagogical knowledge. Although not directly generalizable to other organizations, this case study and its conclusions and recommendations could be used by others in formulating implementation plans and developing strategies. It does add to the body of case studies and can be useful, as Flyvberg (2007) suggests.

On the other hand, the section has not employed a consistent methodology for estimating and evaluating the benefits from its programmes. Evaluations tend to focus on attitudinal benefits regarding ODL and individual experiences, rather than taking into account the broader array of benefits (realized and otherwise) flowing from its grant work and other initiatives. Finally, the way in which the section's programmes were organized and managed made a real difference to the benefits that were produced by the initiatives undertaken.

To assess the benefits of the first iterations of the eILO programmes, the section engaged evaluators to examine the programmes, to report on the benefits and problems encountered, and to make recommendations. These evaluations can be seen as mini case studies of each programme. The projects were similar and the evaluations tended to cluster around the same set of benefits and recommendations, for example stressing the high quality of the instructional materials and the importance of the tutor-learner relationship. There were sufficient programme evaluations to reliably inform the analytical framework and to draw reliable conclusions about the success or failure of the overall program.

Perhaps the most difficult analytic problem is assigning to the section a proportion of the overall benefit of the programmes in a manner that properly reflects the section's contribution to it. For example, how much of the partners' and learners' input was responsible for the overall success? There is no reliable way to accurately quantify the section's contribution in most cases, and doing so remains a methodological challenge for the future; although, having a pre- and post-test of learner achievement should go a long way towards increasing the reliability of any evaluation. For the purposes of this study, the evaluator has attempted to specify in his analyses the role that the eLIO section has played. Based on this assessment a conservative judgment was used to characterize the section's contribution for purposes of developing findings and recommendations. What cannot be dismissed in any analysis of the work of this section is the fact that all of the 
stated goals were met and in some cases exceeded, coupled with an overwhelming degree of satisfaction expressed by all of the participants, including the learners, the partners, the tutors, the COL staff, and the eLIO section employees.

\section{Recommendations}

Taking into account the general level of satisfaction and increasing number of clients, the following recommendations are suggested:

1. Keep doing what you are doing.

2. Edit and revise the material to model plain English.

3. Re-examine your pricing structure to maximize revenues as much as possible.

4. Negotiate with partners either copyright control or an open license.

5. Implement new technologies to support the learning management and administration (LMS, CRM).

6. Develop a succession plan for the section if the present manager leaves.

\section{Acknowledgements}

The author would like to acknowledge the help of Angela Kwan, the learning manager of eLIO, and her staff as well as the many interviewees who assisted in this case study. 


\section{References}

Clark, R. E. (1983). Reconsidering the research on learning from media. Review of Educational Research, 53(4), 445-459.

Cruickshank, D. R. (1990). Research that informs teachers and teacher educators. Bloomington, IL: Phi Delta Kappa Educational Foundation.

Flyvberg, B. (2006). Five misunderstandings about case-study research. Qualitative Inquiry, 12(2), 219 - 245.

Hills, H. (1999). Lloyds Bank and multimedia. In S. Brown (Ed.), Open and distance learning: Case studies from industry and education. London: Routledge.

Leedy, P. D. (1993). Practical research: Planning and design. New York: Macmillan.

Long, L. K., Janas, D. L., Kay, L., \& August, C. (2009). Introducing online learning at a small college through a faculty learning community. Online Journal of Distance Learning Administration, 12(1).

Mathur, R., \& Oliver, L. (2007). Developing an international distance education program: A blended learning approach. Online Journal of Distance Learning Administration, 10(4).

Nchindila, B. (2007). Conditions for the success of online mentoring: A case study. Online Journal of Distance Learning Administration, 10(2).

Nunan, D. 1992. Research methods in language learning. Cambridge: Cambridge University Press.

Oliveira, L. R. d., Cortimiglia, M., \& Marques, L. F. M. (2003). Administering a web-based course on data technology. Online Journal of Distance Learning Administration, 6(4).

Roth, G., \& Kleiner, A. (no date). The learning history research project. Retrieved from http://ccs.mit.edu/LH/.

Yin, R. (1994). Case study research: Design and methods. London: Sage. 


\section{Appendix}

\section{Reports and Publications Consulted}

Committee on Benefits of DOE R\&D on Energy Efficiency and Fossil Energy. (2001). Energy research at DOE: Was it worth it?

Efficiency and fossil energy research 1978 to 2000. Washington DC: Commission on Engineering and Technical Systems, National Research Council.

Commonwealth of Learning. (1999). Final proposal (UNHCR bid). Vancouver: Commonwealth of Learning.

Commonwealth of Learning. (2002a). Technical proposal (AIDS/WHO). Vancouver: Commonwealth of Learning.

Commonwealth of Learning. (2002b). Technical proposal for writing effectively: A submission to the International Labour Organization. Vancouver: Commonwealth of Learning.

Commonwealth of Learning. (2006). Learning for development: The 2006 -2009 Plan. Vancouver: Commonwealth of Learning.

Commonwealth of Learning. (2007a). Evaluation request for baseline information. Vancouver: Author.

Commonwealth of Learning. (2007b). Completion report: WHO/UNAIDS Cohort \#12: June 15 December 15, 2007. Vancouver: Commonwealth of Learning.

Commonwealth of Learning. (2007c). L4L AK elearninig with IOs: Evaluation plan outline. Vancouver: Commonwealth of Learning.

Commonwealth of Learning. (2007d). Results for WHO June 2007 session. Vancouver: Commonwealth of Learning.

Commonwealth of Learning. (2007e). Submission to CADE Awards of Excellence Programs: "Writing Effectively for UNHCR" course. Vancouver: Commonwealth of Learning.

Commonwealth of Learning. (2007f). Technical proposal for writing effectively for OIOS: A submission to the United Nations Vancouver: Commonwealth of Learning.

Commonwealth of Learning. (2007g). The World Bank HRSLO: Report writing [CDRom]. Vancouver: Author. 
Commonwealth of Learning. (2007h). World Health Organization: Writing effectively for WHO [CDRom]. Vancouver: Author.

Commonwealth of Learning. (2007i). Writing effectively for UNICEF: A Submission to UNICEF (India). Vancouver: Commonwealth of Learning.

Commonwealth of Learning. (2008a). Commonwealth of Learning discussion area: List of listservs. Vancouver: Author.

Commonwealth of Learning. (2008b). International Labour Organization: Writing effectively for the ILO [CDRom]. Vancouver: Author.

Commonwealth of Learning. (2008c). Listserv participation list. Vancouver: Author.

Commonwealth of Learning. (2008d). Monthly progress reports (WHO, UNAIDS ILO, ILO2, Worldbank). Vancouver: Author.

Commonwealth of Learning. (2008e). Pilot: Writing effectively for UNICEF, India country office [CDRom]. Vancouver: Author.

Commonwealth of Learning. (2008f). The World Bank HRSLO: Writing e-mails, memos and letters [CDRom]. Vancouver: Author.

Commonwealth of Learning. (2008g). OIOS course analysis. Vancouver: Author.

Commonwealth of Learning (2008h). Elearning for international organisations. Vancouver: Author.

Commonwealth of Learning. (n. d.-a). Draft only evaluation report: Writing Effectively for UNHCR (Trial Version) Vancouver: Commonwealth of Learning.

Commonwealth of Learning. (n. d.-b). Evaluation request for baseline info. Vancouver: Author.

Commonwealth of Learning. (n. d.-c). Proposal for IFRC. Vancouver: Commonwealth of Learning.

Commonwealth of Learning. (n. d.-d). Writing audit reports for OIOS - Development cost analysis. Vancouver: Author.

Dunlop, C. (2005). Writing effectively - Distance learning course for the International Federation of Red Cross and Red Crescent Societies: Assessing the return on expectations. Vancouver: Research and Evaluation Unit, Simon Fraser University. 
John, K. (2005). Writing effectively for UNHCR: Course evaluation report. Vancouver: Commonwealth of Learning.

Kwan, A. (2002). Staff development: training more with less - a model for international development agencies. Pan-Commonwealth Forum 2. Durban, South Africa.

Kwan, A. (2005a). Comparing learning effectiveness based on use of different media for delivery of content. Pan-Commonwealth Forum 3. Dunedin, South Africa: Commonwealth of Learning.

Kwan, A. (2005b). Elearning for international organisations. Vancouver: Commonwealth of Learning.

Kwan, A. (2005c). Staff development: Training more with less: A model for international development agencies. Pan-Commonwealth Forum 2. Durban, South Africa: Commonwealth of Learning.

Kwan, A. (2006). Business plan for COL programme - "Human resources development through eLearning for international organisations” 2006-2009 (Submitted to senior management meeting, April 2006). Vancouver: Commonwealth of Learning.

Kwan, A. (2008a). Learning for livelihood: Transnational programmes. Vancouver: Commonwealth of Learning.

Kwan, A. (2008b). Learning for livelihoods: Transnational programme (Powerpoint). Vancouver: Commonwealth of Learning.

Kwan, A., \& Martel, A. (2005). Designing distance education for international organisations: Testing theory from a field-attentive approach. Canadian Association of Distance Education Conference, Vancouver.

Kwan, A., \& Smulders, G. (2006). Think globally, learn locally: Democratising learning for international organisations. Pan-Commonwealth Forum 4, Ocho Rios, Jamaica.

Lentell, H., \& O’Rourke, J. (2004). Tutoring large numbers: An unmet challenge. International Review of Research in Open and Distance Learning, 5(1).

Murphy, D. (2003). Evaluation report of a course on writing effectively: Commonwealth of Learning/International Federation of Red Cross and Red Crescent Societies. Hong Kong: Open University of Hong Kong.

O'Rourke, J. (2003). Lost and found: Elearning outside the doors of academe. In A. Tait \& R. Miller (Eds.), Rethinking learner support in distance education: Change and continuity in an international context. London: Routledge. 
Ruhe, V. (2002). A course on writing effectively for UNHCR: Evaluation report. Vancouver: Commonwealth of Learning.

Ruhe, V., \& Zumbo, B. D. (2006). Using Messick’s framework to validate assessment tasks in online environments: A course in writing effectively for UNHCR. In D. D. Williams, S. L. Howell, \& M. Hricko (Eds.), Online assessment, measurement and evaluation: Emerging practices (pp. 203-226). Pennsylvania: Idea Group Inc.

Shale, D. (2002). Evaluation report on WHO/UNAIDS training course - Writing Effectively (Pilot offering September/October, 2002). Calgary: University of Calgary.

Spaven, P. (2006). Evaluation of the 2003-2006 Commonwealth of Learning strategic plan: Final report. Retrieved from http://www.col.org/SiteCollectionDocuments/0603_Evaluation.pdf.

Trevor-Smith, H. (2008). Transnational programmes (Powerpoint). Vancouver: Commonwealth of Learning.

Walker, R., \& Dzakiria, H. (2004). Tutor's feedback on the course on Writing Effectively for UNHCR/UNAIDS and WHO: Stage 2. Norwich, UK: University of East Anglia.

Walker, R., \& Dzakiria, H. (2005). Impact of the Effective Writing Course in WHO and UNAIDS: Evaluation Study for the Commonwealth of Learning - Stage 3. Norwich, UK: University of East Anglia.

Wilson, M. (2006). Evaluation report: Writing effectively for the ILO. Vancouver: Commonwealth of Learning.

Wilson, M. (2007). World Bank Report Writing distance learning course: Evaluation report. Vancouver: Commonwealth of Learning.

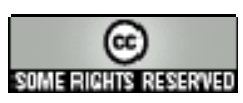

